\title{
Tocquevilles „Demokratie in Amerika“ und die gesellige Gesellschaft seiner Zeit
}

Es entbehrt nicht der Ironie, daß einer der kanonischen Texte der amerikanischen Demokratie von einem französischen Aristokraten verfaßt wurde. Um ihren Argumenten Gewicht zu geben, berufen sich noch heute Liberale wie Konservative in den Vereinigten Staaten auf jenen Reisebericht, den Alexis de Tocqueville in zwei Bänden 1835 und 1840 veröffentlicht hat. Nicht nur Politiker, sondern auch viele Sozial- und Politikwissenschaftler gehen von einer ungebrochenen Gültigkeit der Thesen Tocquevilles aus. Hierzu gehört seine Überzeugung, daß die Grundlage der amerikanischen Demokratie auf ihren geselligen Vereinigungen beruhe. Mit Bewunderung hatte Tocqueville beobachtet, wie sich die Bürger in den Vereinigten Staaten - anders, wie er meinte, als in Kontinentaleuropa - in unzähligen Vereinen engagierten und so die Demokratie mit Leben füllten.

Um so alarmierender erschien in den Augen des Politikwissenschaftlers Robert Putnam das erste Ergebnis seiner empirisch-statistischen Erhebungen, die er 1995 im „Journal for Democracy“ unter dem Titel „Bowling Alone“ veröffentlichte. Obwohl mehr Amerikaner zum Kegeln gehen als je zuvor, habe der Anteil derjenigen erheblich abgenommen, die es im Verein tun. Auch die Mitgliedschaft in so unterschiedlichen Vereinigungen wie den Boy Scouts, dem Roten Kreuz oder den Freimaurerlogen sank dramatisch in den letzten vierzig Jahren, ebenso die Teilhabe der Bürger an den Angelegenheiten ihres lokalen Gemeinwesens. Nur nationale Organisationen wie die „Amerikanische Vereinigung der Rentner", die allein der Interessenvertretung dienen und kein gemeinsames geselliges Leben besitzen, scheinen nach wie vor zu florieren. ${ }^{1}$ Die Amerikaner gehen heute nicht mehr gemeinsam kegeln, sie sehen alleine fern oder surfen im Internet und lassen ihre Interessen von Organisationen vertreten, mit denen sie nur brieflich im Kontakt stehen. Ohne bürgerschaftliches Engagement in geselligen Vereinen aber keine Demokratie, sondern eine „,couch potato democracy“ - so könnte man Putnams Befürchtung zusammenfassen. Selbst die häufig als Gegenargument angeführte zunehmende Bereitschaft, für gemeinnützige Zwecke zu spenden oder zu stiften, könne kein Ersatz für ein geselliges Leben sein. Denn „soziales Kapital“, also bestimm-

1 Vgl. Putnam 1995; Putnam 1996; sowie jetzt Putnam 2000. 
te meßbare Ressourcen des Zusammenlebens, die auf sozialen Netzwerken, Normen und Vertrauen aufbauten, sei für die Demokratie bedeutsamer als das altruistische Handeln einzelner.

Mit dieser These vom Schwund des sozialen Kapitals am Ende des 20. Jahrhunderts, das die Bürger seit den Anfängen der amerikanischen Demokratie in geselligen Vereinen akkumuliert hätten, ist Putnam in den Vereinigten Staaten praktisch über Nacht zu einer Figur des öffentlichen Lebens geworden. „Bowling Alone“ wurde bald zu einem ähnlichen Schlagwort wie mehr als vierzig Jahre zuvor David Riesmans „Lonely Crowd" - ebenfalls ein sozialwissenschaftlicher Bestseller, der die Vereinzelung und „Außensteuerung" der Amerikaner als Problem für das politische Gemeinwesen begriff. Putnam, dessen Bücher bis dahin über die akademische Welt hinaus niemand kannte, galt seit „Bowling Alone“ als ein gern gesehener Gast sowohl in TV Talk Shows als auch in Camp David. ${ }^{2}$ Im letzten Präsidentschaftswahlkampf suchten beide Kandidaten das Gespräch mit dem Harvard Professor über die Frage, wie die Amerikaner zu einer „Reinvestition“ in soziales Kapital bewegt werden könnten. Und seine Thesen haben nicht nur für Diskussion gesorgt, sondern auch großangelegte quantifizierende Forschungsprojekte provoziert (eines geleitet von Putnam selber, ein anderes von seiner Harvard Kollegin Theda Skocpol), deren zum Teil sich widersprechende Ergebnisse jetzt vorliegen. ${ }^{3}$

Die Frage, ob die Amerikaner sich heute zahlenmäßig tatsächlich weniger an geselligen Vereinen beteiligen, soll im Folgenden nicht weiter verfolgt werden. Vielmehr sollen einige Grundannahmen sowohl Putnams als auch seiner Kritiker, die sich alle auf Tocqueville und sein Bild der amerikanischen Gesellschaft berufen, aus historischer Perspektive geprüft werden, Grundannahmen, die dem empirischen Befund Putnams überhaupt erst ihre vermeintliche politische Bedeutung geben. Es geht mithin um den Versuch, Tocquevilles These eines Zusammenhangs von Assoziation und Gemeinsinn, die für die amerikanische Demokratie selbst zu einem Glaubenssatz geworden ist, zu historisieren. Zunächst soll deshalb die Argumentation im entsprechenden Kapitel von „Demokratie in Amerika“ noch einmal skizziert werden. Worin lag für Tocqueville die überragende Bedeutung geselliger Vereine in der Demokratie? Wie erklärt sich sein leidenschaftliches Plädoyer für eine politische Wissenschaft, die sich vornehmlich mit den Assoziationen beschäftigen sollte? In einem zweiten Schritt soll das, was man die „gesellige Gesellschaft" des 18. und 19. Jahrhunderts nennen könnte, Tocquevilles Thesen gegenübergestellt werden. Läßt sich seine Behauptung halten, die Verbreitung und Popularität der geselligen Vereine sei eine Besonderheit der amerikanischen Gesellschaft und Demokratie gewesen? Sind Tocquevilles Thesen eingebettet in einen gemeineuropäisch-transatlantischen Diskurs und daran gebundene soziale Praktiken seiner Zeit? Schließlich sollen abschließend Tocquevilles Thesen (und auch die seiner heutigen Adepten) an der ambivalenten Geschichte dieser ,geselligen Gesellschaft" gemessen werden. Können, historisch gesehen, die geselligen Vereine als Garanten einer le-

2 Putnam 2000, S. 509. Schon in seiner Studie über das Demokratiegefälle zwischen Nord- und Süditalien hatte Putnam betont, daß demokratische Institutionen auf der historischen Tradition bürgerschaftlichen Engagements etwa in Vereinen beruhen. Putnam 1993, S. $89 \mathrm{ff}$.

3 Putnam 2000; Skocpol/Fioring 1999; Skocpol 1997. 
bendigen Demokratie gelten, oder verlangt die Berufung auf Tugend und Gemeinsinn immer auch den elitären Ausschluß und die politisch-moralische Maßregelung jener, die nicht als tugendhaft gelten?

1. Tocqueville handelt von den Assoziationen in beiden Teilen von "Demokratie in Amerika", allerdings auf verschiedene Art und Weise. Im ersten Teil, der eine Analyse der politischen Ordnung der Vereinigten Staaten bietet, spricht Tocqueville den Vereinen jene Bedeutung zu, die heute vertraut klingt und bei Putnam und anderen mitschwingt. Zur Lösung ihrer sozialen und politischen Probleme wenden sich die Amerikaner nicht an eine Obrigkeit, sondern gründen einen Verein. Sie nehmen mithin ihr Leben in die eigene Hand und wirken für das Gemeinwohl. Selbst für einen in den Augen eines französischen Aristokraten befremdlichen Zweck wie die Bekämpfung der Trunksucht bilden sich in Amerika sofort unzählige Assoziationen. Dementsprechend gehört die Vereinigungsfreiheit, mehr noch als die Pressefreiheit, zu den wichtigsten Rechten in der Demokratie und wenn sie auch nicht ohne politische Gefahren ist, erscheint sie Tocqueville doch als das Mittel, um eine noch größere Gefahr, die in der Demokratie droht: die politische Tyrannei der Mehrheit, zu bannen. ${ }^{4}$

Es hieße aber Tocquevilles Anliegen mißzuverstehen, wolle man in ihm einen frühen Soziologen politischer Verfassungsordnungen und in seiner Aufmerksamkeit für die Vereine nur ein Plädoyer für mehr bürgerschaftliches Engagement und intermediäre Gewalten sehen. Tocqueville stand der Demokratie skeptisch, dem beginnenden soziologischen Denken seiner Zeit nahezu feindlich gegenüber, woran Wilhelm Hennis vor zwei Jahrzehnten in einem fulminanten Essay erinnert hat. Tocqueville suchte nach einem Weg, die Trennung von Mensch und Bürger, von Individualität und Sozialität zu verhindern. „Für alles wahrhaft politische Denken“, so Hennis, „ist das Verhältnis von Mensch und Bürger das zentrale politische Problem, für das soziologische Denken ist das ein Nicht-Mehr-Problem." Hierin liegt auch die Differenz zu seinem jüngeren Zeitgenossen Karl Marx. „Tocqueville konnte sich, realistischer als Marx, die Erledigung dieses Problems nur in Gestalt der egalitär-demokratischen Tyrannis vorstellen. Diese Form der Erledigung des Problems zu verhindern, war die Triebkraft seines leidenschaftlichen denkerischen Versuchs. “5

Es ist jene Tradition der klassischen politischen Theorie, die stets nach den Auswirkungen einer Regierungsform auf den von ihr geformten Menschentypus, auf seine Tugend, fragt und daran ihren Wert bemißt, an die Tocqueville anzuknüpfen suchte. Es ging ihm mithin nicht allein, ja nicht einmal in erster Linie, um die politische Verfassung eines Gemeinswesens, sondern um die „Seelenverfassung“ der Bürger, die dieses Gemeinwesen hervorbringt, um jene sozial-moralischen Grundlagen politischer Ordnung, die in der jüngeren politischen Theorie bestenfalls als „vorpolitisch" gelten. ${ }^{6}$ Tocqueville hielt die Gefühle der Menschen, ihre innere Verfaßtheit, für grundlegender für ihr Zusammenleben als ihre rational bedachten Rechte und Interessen. Er war davon

\footnotetext{
4 Tocqueville [1835] 1987, S. 285.

5 Hennis 1982, S. 390; sowie Aron 1981.

6 Vgl. die kritischen Bemerkungen bei Münkler 1996, S. 8; sowie allg. Münkler 1991; Münkler 1992.
} 
überzeugt, „daß die politischen Gesellschaften nicht das Produkt ihrer Gesetze sind, sondern schon von vornherein durch die Gefühle, Glaubensarten, Ideen und Herzensund Geistesangelegenheiten der Menschen, die in ihnen sind, bestimmt werden ". ${ }^{7}$ Tocqueville teilte als „aristokratischer Liberaler" mit seinen Zeitgenossen John Stuart Mill oder Jacob Burckhardt die Skepsis gegenüber dem heraufziehenden demokratischen Zeitalter. ${ }^{8}$ Er sah sich, in den Worten von Hennis, als eine Art "Seelenhistoriker", als ein „Analytiker der Ordnung und Unordnung der menschlichen Seele im demokratischen Zeitalter". ${ }^{9}$ Die alles entscheidende Frage lautete für ihn, wie eine seelische Verarmung, die dem Menschen gerade in den demokratischen Gesellschaften droht und die dem Despotismus ein Einfallstor bietet, verhindert werden könne.

Eine Antwort auf diese Frage meinte Tocqueville mit den geselligen Vereinen gefunden zu haben. Entsprechend finden sich die entscheidenden Passagen zur Bedeutung der Assoziationen im zweiten Teil seines Buches, der vom Einfluß der Demokratie auf das geistige und sittliche Leben, hier insbesondere auf das Gefühlsleben der Amerikaner und abschließend umgekehrt von dessen Auswirkungen auf die politische Gesellschaft handelt. „Nur durch die gegenseitige Wirkung der Menschen aufeinander“, formuliert Tocqueville den Grundsatz seines politischen Denkens, ,erneuern sich die Gefühle und die Gedanken, weitet sich das Herz und entfaltet sich der Geist der Menschen." Diese Wechselwirkung, die in der ständischen Gesellschaft festen Regeln unterlag, müsse in der bürgerlichen Gesellschaft künstlich hervorgerufen werden. ${ }^{10}$ „Und das allein können die Vereinigungen tun. " ${ }^{11}$ Nichts verdiene eine größere Aufmerksamkeit, heißt es nun apodiktisch, als die rein geselligen Vereine, welche den Geist und die Sitte heben und das Gefühlsleben bereichern. Jene erscheinen ihm sogar bedeutsamer als die Vereine zu unmittelbar politischen oder gewerblichen Zwecken, die Tocqueville im ersten Teil von „Demokratie in Amerika" abgehandelt hatte und die, wie er jetzt schreibt, uns leichter auffallen, während die anderen uns entgehen. Die scheinbar unpolitischen, nicht von partikularen Interessen geleiteten Vereine entreißen den Einzelnen seiner selbstsüchtigen Schwäche und knüpfen in der egalitären, anomischen Gesellschaft neue Bande, jene liens, die im politischen Denken Tocquevilles eine überragende Bedeutung einnehmen. „Unter den Gesetzen, denen die menschlichen Gesellschaften unterstehen“, so weiter Tocqueville, ,gibt es eines, das genauer und klarer erscheint als alle andern. Damit die Menschen gesittet bleiben oder es werden, muß sich unter ihnen die Kunst der Vereinigung in dem Grade entwickeln und vervollkommnen, wie die gesellschaftlichen Bedingungen sich ausgleichen. “12 Das bedeutet umgekehrt auch: In dem Maße, wie sich das

7 Brief vom 26.10.1853, zit. n. Hennis 1982, S. 395.

8 Kahan 1992.

9 Hennis 1982, S. 402.

10 „Die Menschen sind hier nicht mehr durch Kasten, Klassen, Korporationen und Geschlechter miteinander verbunden und sind daher nur zu sehr geneigt, sich bloß mit ihren besonderen Interessen zu beschäftigen, immer nur an sich selbst zu denken und sich in einen Individualismus zurückzuziehen, in dem jede öffentliche Tugend erstickt wird." Tocqueville [1856] 1978, S. 15.

11 Tocqueville [1840] 1987, S. 164. Zu Tocquevilles „Demokratie in Amerika“ vgl. u.a. Jardin 1981, S. 93-252; Marshall/Drescher 1968; Wilentz 1988; Kloppenberg 1996.

12 Tocqueville [1840] 1987, S. 166f. 
verbindende Band der Individuen, das ihre Tugend garantiert, lockert, erodieren die politischen Grundlagen des demokratischen Gemeinwesens. Je weniger sich die Bürger in der l'art de s'associer, der Kunst der geselligen Vereinigung, üben, desto mehr werde das Durchschnittsniveau der Herzen und Geister sinken und sich Gleichheit und Despotismus unheilvoll verbinden.

Wie eine demokratische Gesellschaft aussieht, die sich ihrer politischen Grundlagen nicht mehr in der Geselligkeit ihrer Bürger versichert, hat Tocqueville mit einem apokalyptischen Bild beschrieben:

\begin{abstract}
„Ich erblicke eine Menge einander ähnlicher und gleichgestellter Menschen, die sich rastlos im Kreise drehen, um sich kleine und gewöhnliche Vergnügen zu verschaffen, die ihr Gemüt ausfüllen. Jeder steht in seiner Vereinzelung dem Schicksal aller anderen fremd gegenüber: seine Kinder und seine persönlichen Freunde verkörpern für ihn das Menschengeschlecht; was die übrigen Mitbürger angeht, so steht er neben ihnen, aber er sieht sie nicht; er berührt sie, und er fühlt sie nicht; er ist nur in sich und für sich allein vorhanden, und bleibt ihm noch eine Familie, so kann man zumindest sagen, daß er kein Vaterland mehr hat. Über diesen erhebt sich eine gewaltige, bevormundende Macht, die allein dafür sorgt, ihre Genüsse zu sichern und ihr Schicksal zu überwachen. Sie ist unumschränkt, ins einzelne gehend, regelmäßig, vorsorglich und mild. Sie wäre der väterlichen Gewalt gleich, wenn sie wie diese das Ziel verfolgte, die Menschen auf das reife Alter vorzubereiten; statt dessen aber sucht sie bloß, sie unwiderruflich im Zustand der Kindheit festzuhalten. " 13
\end{abstract}

Die Geselligkeit besitzt, so könnte das Argument Tocquevilles zusammengefaßt werden, in der Demokratie eine herausragende politische Bedeutung, indem sie jene Bande zwischen den Menschen neu knüpft, welche die heraufziehende demokratische Gesellschaft zunächst zerstört, indem sie die alte Ordnung auflöst und die Selbstsucht der Menschen befördert. Die „neue politische Wissenschaft“, die Tocqueville als „Grundwissenschaft der bürgerlichen Gesellschaft" begründen wollte, sollte sich deshalb vorrangig mit der Kunst der Vereinigung - der Geselligkeit - beschäftigen. Von ihrem Fortschritt hänge, wie er pathetisch, aber wissenschaftsgeschichtlich folgenlos schreibt, der Fortschritt aller anderen Wissenschaften ab. ${ }^{14}$ Es ist dieses leidenschaftliche, grundsätzliche Verständis politischer Wissenschaft und des Zusammenhangs von Assoziation und politischer Tugend, das selbst jenen in der Gegenwart fehlt, die sich auf Tocqueville berufen und den zahlenmäßigen Rückgang von geselligen Vereine beklagen. Wenn Putnam meint, die Zugehörigkeit zu Vereinen erhöhe die Lebensqualität der Bürger, dann hat er äußere, praktische Vorteile für Nachbarschaften mit hohem ,sozialen Kapital" im Blick: ein höheres Einkommen durch das Knüpfen von Geschäftskontakten, bessere Bildung und Gesundheit, mehr Sicherheit und eine niedrigere Kriminalitätsrate - nicht aber die innere, ,seelische“ Qualität der Bürger, ihre Tugend.

13 Tocqueville [1840] 1987, S. 463. Noch im Vorwort von „Der alte Staat und die Revolution" bemerkt Tocqueville trocken, daß auch zwanzig Jahre nach dem Erscheinen von „Demokratie in Amerika" in der Welt nichts geschehen sei, das ihn veranlaßt habe, anders zu denken und zu sprechen. Die Demokratie berge weiterhin die Gefahr des Despotismus. Tocqueville [1856] 1978, S. 15.

14 Tocqueville [1840] 1987, S. 166. Vgl. Hennis 1982, bes. S. $396 \mathrm{ff}$. 
II. So fremdartig der von Tocqueville behauptete politische Zusammenhang von Assoziation, Gemeinsinn und Bürgertugend heute wirken mag, so vertraut war er den „Praktikern der Bürgergesellschaft“ im 18. und 19. Jahrhundert. ${ }^{15}$ Das ist die These, die im Folgenden entfaltet werden soll. Weder stand Tocqueville mit seinen Ansichten allein, noch war die Hochschätzung und Verbreitung der geselligen Vereine eine Besonderheit der amerikanischen Gesellschaft. Die Betonung von Assoziation und politischer Tugend kann vielmehr als Teil eines gemeineuropäisch-transatlantischen Diskurses und von daran gebundenen Praktiken von der Mitte des 18. Jahrhunderts bis zum Ersten Weltkrieg angesehen werden. ${ }^{16}$

Gegen diese These sprechen jeweils ein vertrautes Argument der politischen Ideengeschichte und der Sozialgeschichte. Beide Argumente gilt es zunächst zu entkräften. In der politischen Ideengeschichte herrscht, vereinfacht gesagt, die Auffassung vor, der ältere Klassische Republikanismus und civic humanism mit seiner Betonung der politischen Tugend sei gegen Ende des 18. Jahrhunderts abgelöst worden von einem Glauben an den Fortschritt und die Verfolgung von jeweils eigenen Interessen, die sich in einem politischen und wirtschaftlichen Gemeinwesen letztlich ausgleichen und diesem Stabilität verleihen würden. Der Tugenddiskurs der aristotelischen Tradition wird aber mit der Aufklärung und im frühen Liberalismus nur umgewandelt zur Idee der Verbesserung des einzelnen, seiner inneren Verfaßtheit, in der Geselligkeit der Bürger - auch und gerade aufgrund des Erfahrungsumbruchs und des Krisengefühls vor und nach den Revolutionen des späten 18. Jahrhunderts. „For Revolutionary Americans sensibility and sociability became modern surrogates for the classical virtue that theorists for millennia had thought necessary for sustaining a republican government", wie Gordon Wood prägnant formuliert hat. „Some substitute for this ancient martial virtue had to be found, and many discovered it in what was increasingly perceived as the natural sociability, sentimentality, and politeness of people. ${ }^{\text {“17 }}$ Wie vielfältig auch die Formen waren, welche die englischsprachige und die kontinentaleuropäische Aufklärung im 18. Jahrhundert annahm, sie verblieb hier wie dort im Deutungshorizont der aristotelischen Tradition, indem sie in der „ungeselligen Geselligkeit“ (Immanuel Kant) des Menschen, seinem Hang, sich zu vereinzeln und seinem Drang, sich zu vergesellschaften, eine anthropologische Begründung bürgerlicher Gesellschaft suchte. Im geselligen Austausch mit anderen sollten sich die Menschen jene sozialen Tugenden aneignen, die sie als Bürger eines politischen Gemeinwesens benötigten. Die Geselligkeit versprach, wie unzählige Male nicht nur von den Theoretikern, sondern auch von den weniger bekannten Praktikern der Bürgergesellschaft formuliert wurde, ,mutual improvement, for increasing our knowledge, and mending our hearts". ${ }^{18}$ Auch Republikanismus und Liberalismus, von der politischen Ideengeschichte unnötig scharf voneinander geschieden, verschmelzen historisch in dieser Auffassung von der natürlichen Sozialität des Menschen und seiner Fähigkeit, sich im Austausch mit anderen Tugend und Gemeinsinn anzueignen, das eigene Selbst zu bilden

15 Der Begriff „practitioners of civil society“ nach Hull 1995, S. 2.

${ }^{16}$ Der Verfasser bereitet zu diesem Thema eine transnational vergleichende Studie vor.

17 Wood 1999; sowie allg. Wood 1991.

18 T. Burges, Solitude and Society Contrasted, Providence 1797, S. 19, zit. n. Clark 2000, S. 413; sowie u.a. Klein 1998; Klein 1994; Mullan 1988; Gordon 1994. 
und zu regieren, gegen den als ,modern“ und beherrschend wahrgenommenen Trend einer zunehmenden Verfolgung nur partikularer Interessen, der das politische Gemeinwesen moralisch korrumpiere. ${ }^{19}$

Darum ging es, wie gesehen, Tocqueville; aber er stand damit keineswegs allein. Das zeigt etwa das Beispiel seiner südwestdeutschen Zeitgenossen Carl von Rotteck und Carl Theodor Welcker und die Artikel „Association“" „Gemeinsinn" und „Bürgertugend" in dem von ihnen herausgegebenen "Staatslexikon". 20 "Freie Associationen" gelten dort als „die Quelle aller höheren Menschlichkeit und Cultur" und werden anthropologisch im „Geselligkeitstrieb“ und göttlich, in der Macht der Vorsehung gegründet.

\begin{abstract}
„Denn während andere Geschöpfe ihre Bedürfnisse befriedigen, sich schützen und ihre Bestimmung erreichen können, ohne verschiedenerlei gesellschaftliche Verbindungen, erhalten sich die Menschen erst durch die mannigfachsten, je nach Zeit, Ort und Verhältniß verschiedenen Verbindungen, durch wechselseitigen Austausch und Verein ihrer Einsichten, Erfahrungen und Kräfte, und in denselben ihre höhere Entwicklung und die nöthigen Antriebe und Mittel zu allen reichen und großen Aufgaben ihrer Bestimmung. ${ }^{.21}$
\end{abstract}

Ähnlich wie Tocqueville galten auch Rotteck und Welcker die Assoziationen als ein Weg, den Menschen aus seiner Selbstsucht und Vereinzelung herauszuführen. Folglich sahen auch sie den „Gemeingeist" oder „Gemeinsinn" als die ,schönste Frucht des Associationsgeistes" an. ${ }^{22}$ Nicht die Verfolgung der eigenen selbstsüchtigen Interessen, sondern die Selbstverleugnung und der Wille, diese Interessen dem Gemeinwohl unterzuordnen, werden als wahre Tugend beschrieben. Noch prägnanter formuliert es der Artikel zu „Bürgertugend" und „Bürgersinn". „Alle politische Kunst und Verfassung", heißt es dort, ,alle Weisheit für eine gerechte und glückliche Bestimmung und Erhaltung der bürgerlichen Gemeinwesen, der bürgerlichen Verhältnisse und Rechte ist umsonst, ohne Bürgertugend, ohne das, was ihre beiden Hauptbestandteile sind: Bürgersinn und Bürgermuth. Sie bilden die gesunde Lebenskraft der bürgerlichen Vereine. Diese erkranken und ersterben ohne sie. ${ }^{\text {"23 }}$ Die Bürgertugend wird auf dieselbe Weise befördert wie die Tugend überhaupt „durch geistige und sittliche Entwicklung, Erziehung und Uebung; durch Aufklärung, Hervorbildung und Kräftigung der sittlichen Triebe und durch Unterordnung der selbstischen und unsittlichen unter die sittlichen." Tugendübung und Assoziation gehören in der bürgerlichen Gesellschaft zusammmen; dagegen führe - und hierin unterscheiden sich die beiden badischen Liberalen von dem Aristokraten Tocqueville - der Absolutismus zu einer sittlichen Erkrankung der Bürger: ihre Tugend werde krank und faul. „Die Vorherrschaft von Selbstsucht und Sinnlichkeit, Feigheit und Feilheit der Mehrzahl der Bürger und vollends der Beamten war noch immer und überall die verderbliche Folge des Despotismus. “24

\footnotetext{
19 Kahan 1992, S. 5f.; ähnlich z.B. auch Howe 1997, S. 10ff.; vgl. dagegen z.B. Appleby 1992; sowie natürlich Pocock 1975; u. insbes. Pocock 1989; als Überblick Rodgers 1992.

${ }^{20} \mathrm{Vgl}$ allg. hierzu Nolte 1992a; Nolte 1992b; Nolte 1994; sowie Langewiesche 1997.

21 Welcker 1835, S. 21, 23.

${ }^{22}$ Rotteck 1838 , S. 448.

${ }^{23}$ Welcker 1846, S. 748.

${ }^{24}$ Welcker 1846, S. $749 f$.
} 
Beispiele für eine solche Verschmelzung von klassisch-republikanischen und aufgeklärt-liberalen Argumentationsfiguren lassen sich für die Dekaden um 1800 beliebig vermehren und zwar, was oft übersehen wird, über die Grenzen der sich herausbildenden Nationalgesellschaften hinweg. Erinnert sei nur an die Freimaurerlogen des 18. und 19. Jahrhunderts, ohne Zweifel ein gemeineuropäisch-transatlantisches Phänomen. Neuere Studien zeichnen ein grundlegend anderes Bild der Logen, als es noch aus den einflußreichen Studien von Reinhart Koselleck oder François Furet vertraut ist. ${ }^{25}$ Insbesondere an der Interpretation des für heutige Betrachter so bizarren Geheimkults der Logen lassen sich die Unterschiede pointiert zeigen. Die aufklärerische Moral der Freimaurer galt Koselleck und Furet in der Nachfolge von Carl Schmitt und Augustin Cochin als Emanzipationsideologie des recht- und machtlosen Dritten Standes, der sich in den Logen zum Sturz der alten Ordnung versammelte. Hieraus erkläre sich auch der Geheimkult der Freimaurer, die Selbstüberhebung und Verschwörung der Moral gegen die Politik des absolutistischen Staates. Eine solche Sichtweise geht aber, wie neuere Studien zu den westeuropäischen und russischen Freimaurern übereinstimmend zeigen, an ihrem Selbstverständnis und ihren sozialen Praktiken vorbei; ganz abgesehen davon, daß sie die Popularität der Logen im englischsprachigen Raum nicht erklären kann ${ }^{26}$ In Kontinentaleuropa zogen die Logen gleichermaßen aufstrebende Bürgerliche wie aufgeklärte Adlige an, die sich gemeinsam nach unten gegenüber dem ,gemeinen Volk“ abgrenzten. Die vom Geheimnis umschlossenen Logenräumen waren mithin keine Treffpunkte einer aufgeklärten Gegenelite zum absolutistischen Staat, sondern „Stätten des sozialen Kompromisses““ 27

Warum aber dann der Geheimkult der Logen? Das Geheimnis sollte einen Ort in der Gesellschaft schaffen, der nicht geheim, sondern nur geschützt war, um der Tugend dem Leitbegriff der Freimaurer des 18. und 19. Jahrhunderts - einen künstlichen Raum der Entfaltung zu geben. Hieraus erklärt sich auch die Popularität, die nicht nur die Logen, sondern seit den vierziger Jahren des 19. Jahrhunderts allgemein die geheimen Gesellschaften in der Vereinigten Staaten besaßen. Das war auch Tocqueville entgangen, der die geheimen Gesellschaften, ähnlich wie später Koselleck oder Furet, als eine Folge des kontinentaleuropäischen Gegensatzes von Staat und Gesellschaft ansah. Warum sollte es in einer demokratischen Gesellschaft mit einem schwachen Staat dennoch geheime Gesellschaften geben? Aber auch in Kontinentaleuropa behielt die Freimaurerei die Grundzüge ihres Entstehungszusammenhangs in der politischen Kultur Englands und Schottlands am Ende des 17. Jahrhunderts bei. Die französischen, niederländischen oder deutschen Freimaurerlogen

\footnotetext{
,transmitted and textured the Enlightenment, translated all the cultural vocabulary of its members into a shared and common experience that was civil and hence political. Rather than imagining the Enlightenment as represented by the politics of Voltaire, or Gibbon, or even Rousseau, or worse as being incapable of politics we might just as fruitfully look to the lodges for a nascent political modernity. ${ }^{\text {28 }}$
}

${ }^{25}$ Koselleck [1959] 1989; Furet 1980.

26 Vgl. Jacob 1991; Smith 1995; Smith 1999; Bullock 1996.

27 Roche 1981, S. 115.

28 Jacob 1991, S. 224. 
Freimaurer korrespondierten über staatliche Grenzen hinweg; sie konnten auf Reisen die Logen anderer Städte besuchen und fanden dort oft Anschluß an die lokalen Geselligkeitskreise. In den kosmopolitischen Logen zirkulierte mithin die politischmoralische Sprache der Aufklärung jenseits staatlicher und kultureller Grenzen.

Für die russischen Freimaurer etwa war der Zusammenhang von Tugend, Geselligkeit und einer Verbesserung der Gesellschaft zentral. Die Tugend sollte, ganz im Sinne Tocquevilles, Garant einer politisch nicht korrumpierten Gesellschaft sein. Der Weg zur Tugend führte über die Aneignung von Moral und Sitten, von nravouchenie, etwa in den elaborierten freimaurerischen Ritualen.

\begin{abstract}
"The lodge, therefore, occupied a priviledged place in the social landscape of the public. Its inhabitants claimed both to possess secret knowledge required to attain virtue and to be the personification of virtue. This, less than the danger of state repression, accounts for the main function of Masonic secrecy. For through their actions, the Masons attempted to establish a hierarchy within the public based not on the nobility of one's family, nor on one's rank (chin), status at court, or wealth, but on one's proximity to virtue, having placed themselves at its pinnacle. The Masons saw themselves as engaged in nothing less than the construction of a new man, a man of morals and virtue who possessed the traits necessary for the maintenance of the social order and the betterment of the common weal." ${ }^{29}$
\end{abstract}

Eine solche Betonung des sozialen Werts der Tugend ist keine Besonderheit der Logen, sondern ein gemeinsamer Grundzug der europäischen Aufklärung und des frühen Liberalismus, der gleichsam den Antrieb für die Geselligkeitseuphorie der 18. Jahrhunderts und die „Vereinswut" des 19. Jahrhunderts bildete.

Die Logen sahen sich im 18., aber in einem noch stärkeren Maße im 19. Jahrhundert als „Schulen der Bürgertugend“ im Sinne Tocquevilles. Die Freimaurerei solle bewirken, heißt es etwa in einer süddeutschen Logen-Flugschrift aus dem Jahr 1859, „was weder der Staat, noch die Kirche bewirken kann; durch sie soll innere Tugend und Rechtschaffenheit vermehrt und verbreitet werden." Die bürgerliche Gesellschaft könne die innere Tugend nicht befehlen, ,ohne sich zum Richter der Gesinnungen und Gedanken aufzuwerfen, welches die ärgste Tyrannei, und dem wahren Endzwecke der menschlichen Gesellschaft gerade entgegen sein würde." Deshalb bedürfe es sozialer Räume wie der Logen, in denen an der ,inneren Sittlichkeit" des einzelnen gearbeitet werden könne, ,das Gute, welches die bürgerliche Gesellschaft nicht bewirken kann, zu befördern; Weisheit, Freiheit und Tugend in ihrer wesentlichen Reinheit zu erhalten; die Trennungen und Spaltungen, welche das Interesse der Staaten, Religionen, Stände und aller zufälligen Verhältnisse hervorbringt, zu heben, und die Menschen blos durch das allgemeine Band und unter der Regierung des Vernunftgesetzes wieder zu vereinigen. Nach diesem Gesetz sind wir Menschen - weiter nichts. " ${ }^{30} \mathrm{Daß}$ die Logen im 19. Jahrhundert, dem Zeitalter der Öffentlichkeit, an ihrem Geheimkult festgehalten haben, erklärt sich aus diesem politisch-moralischen Selbstverständnis. Sie wollten in der sich demokratisierenden Gesellschaft einen Ort frei halten von den Konflikten dieser Gesellschaft, in dem die Tugend gelebt werden kann. Die Freimaurerlogen und andere gehei-

29 Smith 1995, S. 35, 37.

${ }^{30}$ Pandora, oder interessante Mittheilungen über alte und neue Freimaurerei, aus dem handschriftlichen Nachlasse eines Geweihten, Stuttgart 1859, S. 38f. 
me Gesellschaften verschwinden mithin - anders als Tocqueville und viele Historiker nach ihm geglaubt haben - nicht im Jahrhundert nach der Aufklärung, sondern gewinnen gerade in Großbritannien und den Vereinigten Staaten, aber auch seit den vierziger Jahren in den deutschen Staaten und Frankreich, schließlich seit den sechziger Jahren auch in Italien oder Österreich-Ungarn und nach 1905 für ein Jahrzehnt sogar in Rußland an neuer Popularität und sozialer Bedeutung. ${ }^{31}$

Das Beispiel der Logen zeigt, daß sich dieses Selbstverständnis und die daran gebundenen sozialen Praktiken im 18. und 19. Jahrhundert auf beiden Seiten des Atlantiks finden. Bei der Freimaurerei handelte es sich um eine Art „gesellige Internationale“, die einen transnationalen Raum von Boston bis St. Petersburg, von Kopenhagen bis Neapel umspannte, der den Austausch von Ideen und Meinungen, Praktiken und Praktikern ermöglichte. Den scharfen Gegensatz zwischen amerikanischer Demokratie und den kontinentaleuropäischen Staatsgesellschaften, führte Tocqueville aber vor allem auf das Fehlen der geselligen Vereine, insbesondere in Frankreich, zurück. Der zweite Teil von „Demokratie in Amerika“ enthält kaum mehr einen Reisebericht, sondern eine vergleichend angelegte politische Theorie der bürgerlichen Gesellschaft. Nach Abschluß des Manuskripts fiel auch Tocqueville selber auf, daß es ihm mehr um die Frage der allgemeinen Auswirkungen der Gleichheit auf den Geist, die Gefühle und die Sitten gegangen sei als um ihre spezifischen Erscheinungsformen in der amerikanischen Gesellschaft. ${ }^{32}$ Sein Blick auf die amerikanische Gesellschaft war der eines französischen Aristokraten, der die Gefahren der in seinen Augen unausweichlich kommenden Demokratie für die alteuropäische Gesellschaftsordnung analysierte. Tocqueville entging aus diesem Blickwinkel, in welchem Maße das Assoziationswesen diese Gesellschaftsordnung auf dem europäischen Kontinent eben zu jener Zeit umzuwandeln begann, als er „Demokratie in Amerika" niederschrieb. Als Angehörigem der hauptstädtischen Aristokratie blieben ihm die Verkehrskreise der lokalen bürgerlichen Gesellschaft der französischen Provinz fremd, wo die geselligen Vereine großen Zuspruch fanden. Während Tocquevilles Interesse ganz dem Staat galt, entging ihm das wesentliche Charakteristikum geselliger Vereine: ihre Verankerung in der lokalen Gesellschaft. „Gentlemen's clubs, choral groups, learned societies and other associations were all predominantly provincial“", wie Carol Harrison gezeigt hat. ,In the case of associative sociability, Paris was not the best vantage point for the observation of French society. ${ }^{633}$ Das oft restriktive Vereinsrecht gibt Auskunft über die Haltung des Staates gegenüber den geselligen Vereinen seiner Bürger, nicht aber über das tatsächliche Ausmaß der städtischen Geselligkeit. Im gesamten 19. Jahrhundert standen sich nicht nur in Frankreich, wie Maurice Agulhon festgestellt hat, eine vereinigungsfreudige Gesellschaft und ein vereinigungsfeindlicher Staat gegenüber. ${ }^{34}$ Daraus folgt der ironische Umstand, daß die Tätigkeit der geselligen Vereine in Kontinentaleuropa ungleich besser dokumentiert ist als in den Vereinigten Staaten - in den Akten der staatlichen Behörden, welche die Vereine und cercle mißtrauisch beobachteten.

31 Vgl. u.a. Nord, 1991; Nord 1995: Kap. 1: Freemasonry; Hoffmann 2000.

${ }^{32}$ Marshall/Drescher 1968, S. 523f.

${ }^{33}$ Harrison 1996: 41f.; ausführlich Harrison 1999.

34 Agulhon 1977. 
Der gebannte Blick auf den Staat versperrte Tocqueville (wie auch, für die politische Theorie der „Bürgerlichen Gesellschaft“ ungemein folgenreich, Hegel und Marx), die Wahrnehmung der Bedeutung der Assoziationen für die europäischen Gesellschaften seiner Zeit. Wie im Falle der Logen knüpften auch die geselligen Vereine an die Sprache und die Praktiken der politischen Kultur Englands an. Besaß England schon im 18. Jahrhundert außer den bekannten coffee houses eine Vielzahl von Klubs und Vereinen (in einer Stadt wie Norwich war 1750 jeder fünfte Mann ein Vereinsmitglied), verbreiteten sich diese in Neuengland und auf dem Kontinent seit dem Ende des 18. Jahrhunderts. ${ }^{35}$ Auf beiden Seiten des Atlantiks dienten die Assoziationen als soziale Laboratorien der bürgerlichen Gesellschaft nach vorangegangen politischen Umbrüchen und Krisen. In einer ersten transnationalen Welle der Assoziationsgründung bildete sich insbesondere in französischen und deutschen städtischen Gesellschaften ein dichtes Geflecht an geselligen Vereinen (das erst seit kurzem genau untersucht ist), zeitgleich mit den Vereinigten Staaten, wo die Jahre zwischen 1825 unf 1845 allgemein als die „era of associations" (Mary Ryan) gelten. ${ }^{36}$ Moral improvement und Bildung, obrazovanie und émulation waren die nationalsprachlichen Äquivalente für den von den geselligen Vereinen erhobenen politisch-moralischen Anspruch: Die Verbesserung des Selbst in der Wechselwirkung mit anderen sollte Bürgersinn und, darüber hinweggreifend, Weltbürgersinn und allgemein Humanität bezeugen und bekräftigen. Oft war dieser Anspruch christlich eingefärbt. Nicht nur für Tocqueville enthielt der Zusammenhang von Assoziation, Gemeinsinn und Tugend seinen tieferen Sinn vor dem Hintergrund einer christlichen Brüderlichkeitsethik. ${ }^{37}$ Nur wer im Verein sich selbst, seine Gedanken und Gefühle regieren lerne, könne auch andere regieren. Im geselligen Verein sollte, ähnlich wie in der Loge, an der individuellen Tugend wie am Gemeinwohl gearbeitet werden, beides vereint im harmonischen Ideal der „klassenlosen Bürgergesellschaft“ (Lothar Gall), welches so typisch für den Liberalismus jener Zeit war. ${ }^{38}$ Nicht nur die amerikanischen, sondern auch die französischen oder deutschen Bürger des frühen 19. Jahrhunderts verstanden Interessen als unaufhebbar partikular und zerstörerisch. Nur wer von seinen eigenen Interessen absehen könne, öffne sich, seine „Seele“, im Verein mit anderen und sichere so den Zusammenhalt der Gesellschaft der Bürger. ${ }^{39}$ Die geselligen Vereine, zu jener Zeit sozial exklusiv nur gebildeten und besitzenden Männern offenstehend, sollten ein Gegengewicht zu den Konflikten in Beruf, Familie und Politik bilden. ${ }^{40}$ Sie dienten sicherlich auch dem Amüsement, ja, verschafften diesem einen sozial respektablen Rahmen. In den sozialen Räumen der Vereine erfuhr sich die gesellige Gesellschaft als Gesellschaft, wurden zivile Werte und Tugenden eingeübt und

35 Vgl. zum Folgenden grundlegend: Nord 2000; sowie für England zuletzt: Clark 2000.

36 Vgl. Ryan 1981, S. 105; Ryan 1997, S. 58-93; Blumin 1989, S. 192-229; Gilkeson Jr. 1986; aus der Fülle der Literatur zu Frankreich und den deutschen Staaten: Agulhon 1977; Agulhon 1984; François 1986; Nipperdey 1976; Hardtwig 1994; Hein/Schulz 1996; sowie exemplarisch für viele neuere Studien zum städtischen Bürgertum: Mettele 1998.

37 Vgl. Hennis 1982, S. 396; Kloppenberg 1996, S. 30.

${ }^{38}$ Gall 1976.

39 Reddy 1997: xi; ähnlich Harrison 1999, S. 38; u. Mettele 1998, S. 341.

40 Vincent-Buffault 1995, S. 217. 
anschließend öffentlich gezeigt. Gewiß erfüllten die Vereinen auch unmittelbare soziale oder politische Zwecke, sie verwischten alte Grenzen nach oben und zogen neue Grenzen nach unten - hiervon wird noch die Rede sein. Dennoch kann festgehalten werden, daß die Vereinsseligkeit der Bürger im ersten Viertel des 19. Jahrhunderts, welche die Historiker erst seit kurzem genauer erforscht haben, sich zu einem guten Teil jenem politisch-moralischen Verständnis der Probleme der bürgerlichen Gesellschaft verdankt, die Tocqueville so eindringlich formuliert hat.

Tocquevilles berühmte Passagen in „Demokratie in Amerika“ erscheinen von daher ebensowenig exzeptionell wie die amerikanische Gesellschaft seiner Zeit. Unterstellt man den Praktikern der Bürgergesellschaft des späten 18. und des 19. Jahrhunderts nicht politisch-soziale Ziele, für die sie noch nicht einmal einen Begriff besaßen, öffnet sich ein Zugang zu ihrer eigenen Erfahrungswelt: der weiterhin lebendigen frühneuzeitlichen Tradition politischen Denkens, für die „Bürgertum“ gleichbedeutend mit Tugend und Gemeinsinn war und noch nicht auf eine sozialökonomische Klasse und ihre politische Interessen verwies. ${ }^{41}$ Dadurch werden nicht nur die Spuren des Klassischen Republikanismus im Liberalismus des 19. Jahrhunderts, sondern auch dessen transnationaler Grundzug sichtbar. Wenn man sich von der sozialgeschichtlichen Vorstellung eines engen Zusammenhangs von aufsteigendem Bürgertum als Klasse und Aufklärung und Liberalismus als dessen Emanzipationsideologien löst, wird erkennbar, wie auch in Gesellschaften ohne eine starke „Bourgeoisie“ liberale Ideen und Praktiken (nicht zuletzt die Idee einer moralischen Verbesserung in der Geselligkeit mit anderen) etwa in adligen und gebildeten Kreisen zirkulierten. ${ }^{4{ }^{2}}$

III. „Die Geschichte des Liberalismus läßt sich“, wie Reinhart Koselleck einmal bemerkt hat, „als eine Geschichte des Verzehrs beschreiben. Es ist der Preis, ohne den seine Erfolge nicht zu haben waren. “43 Von einer solchen sich selbst verzehrenden Erfolgsgeschichte zeugt auch die Geschichte der liberalen Begeisterung für gesellige Vereine. Die Vereinseuphorie der ersten Jahrhunderthälfte stellte sich nur als ein Vorspiel für die „Vereinigungswut“, wie es nun hieß, in den beiden Jahrzehnten nach Tocquevilles Tod (1859) heraus. Die gesellige Gesellschaft zwischen Boston und St. Petersburg erlebte in den sechziger und siebziger Jahren einen neuen Aufschwung im Zuge der Überwindung ihrer politisch und sozial je verschiedenen Krisen. Erstmals erfolgte dieser Aufschwung zeitgleich in West und Ost, wo Industrialisierung und Urbanisierung ähnlich tiefgreifende soziale Umbrüche hervorriefen. In den Vereinigten Staaten begann nach dem Bürgerkrieg und der Aufhebung der Sklaverei ein golden age of fraternity, eine sprunghafte Vermehrung geheimer Gesellschaften wie den Odd-Fellows oder den Good Templars. ${ }^{44}$ Die Freimaurerlogen und die christlich geprägten moralischen Reformvereine, die ebenfalls aus Großbritannien kamen, bildeten das kulturelle Vorbild.

\footnotetext{
41 Vgl. Nolte 1992a, S. 628; sowie die semantischen Befunde bei Wirsching 1990; Steinmetz 1991; Koselleck u.a. 1991; Koselleck/Schreiner 1994; Wahrman 1995.

42 So argumentieren z.B. auch polnische Historiker wie Janowski 2000; Jedlinski 1999.

${ }^{43}$ Koselleck o.J., S. 37.

44 Vgl. Carnes 1989; Clawson 1989; Dumenil 1984.
} 
In Kontinentaleuropa ist die Liberalisierung der sich zunehmend als Nationalgesellschaften (oder nationale Teilgesellschaften wie etwa im Falle Österreich-Ungarns) umbildenden Staaten ebenfalls verbunden mit einem bis dahin nicht gekannten Zuwachs an Assoziationen. Wie für die städtische Gesellschaft der französischen Provinz ist auch für die lokale Bürgergesellschaft der deutschen Städte von den Historikern bislang zu wenig wahrgenommen worden, daß das Assoziationswesen sich nach 1860 explosiv ausweitete. Deutschland wird, neben den Vereinigten Staaten, zu dem Vereinsland par excellence. ${ }^{45}$ Mit der massenhaften Zunahme von sozial weniger exklusiven Vereinen wie etwa den Turnern ging der Glauben an einen Zusammenhang von Assoziation und Bürgertugend keineswegs verloren, sondern erfuhr oft eine Übersetzung in eine zeitgemäß scheinende Sprache. So meinte ein Festredner auf einem Dessauer Turnfest im Jahr 1865, um nur ein Beispiel herauszugreifen, im Leben des Vereins erschließe sich „die Vorschule zum Bürgerthum, da blüthen sie auf, die schönsten Bürgertugenden: Selbstbeschränkung, männliche Zucht und Bescheidenheit, da regte sich Freundschaft und Anhänglichkeit, da verwischen sich die enggesteckten Grenzen der Gesellschaft, der Mensch ward zum Menschen und sah auch im Andern den Menschen. ${ }^{\text {“46 }}$

In der Habsburgermonarchie erfolgt 1867 eine Lockerung des Vereinsrecht, die auch hier zu einer sprunghaften Zunahme der Vereinsgründungen führt. In Preßburg (Bratislava/Pozsony) mit einer deutschen, slowakischen und ungarischen Bevölkerung gab es in den fünfziger Jahren nur elf Vereine mit genehmigten Statuten, in den siebziger Jahren ungefähr achtzig mit mehr als 18.000 Mitgliedern. ${ }^{47}$ Vor 1860 hat es nur in wenigen norditalienischen Städten wie z.B. in Mailand Vereine gegeben, jetzt entstand z.B. auch in Neapel ein lebendiges Assoziationswesen. ${ }^{48}$ In den westlichen Provinzstädten Rußlands organisierte sich nach dem Krimkrieg und mit der Ära der „großen Reformen“ die lokale Gesellschaft nun gleichfalls zunehmend in geselligen Vereinen. In einer multiethnischen Stadt wie Odessa gab es zwar schon 1831 einen „Englischen Klub“. Jetzt folgten aber auch ein deutscher Verein „Harmonia“, ein „Wohlgeborenenklub“ und ein jüdischer Verein „Beseda“ - in diesen Klubs und Vereinen traf sich die neue lokale Elite der Stadt aus Kaufleuten, Unternehmern und Beamten. ${ }^{49}$

Zweifellos nahm die Dichte des Vereinswesens von West nach Ost ab. Dennoch überrascht die Ähnlichkeit der Vereinstypen, der Motive der Vereinsgründung und der Wellenbewegungen, mit denen ihre Verbreitung erfolgte. Das zeigt sich insbesondere an der vierten Welle von Vereinsgründungen, die von den neunziger Jahren bis zum Ersten Weltkrieg reicht. In den Ländern, in denen sich schon zuvor ein entwickeltes Assoziationswesen gebildet hatte, explodierte dieses noch einmal zahlenmäßig. Beispielsweise stieg allein die Mitgliedschaft in geheimen Gesellschaften in den Vereinigten Staaten um 1900 auf 5,4 Millionen; nach zeitgenössischen Schätzungen gehörte jeder fünfte Mann einer geheimen Gesellschaften an. Von den insgesamt 275 Assozia-

45 Turner 1994; Tenfelde 1984; die damit beide der geläufigen These vom Niedergang des Assoziationswesens nach 1860 widersprechen.

46 Zit. n. Goltermann 1998, S. 102.

${ }^{47}$ Mannová 1998; allg. Stekl u.a. 1992.

48 Meriggi 1992; Caglioti 1996.

49 Hausmann 2000. 
tionen, die zwischen 1860 und 1914 im nordwestlich von Lyon gelegenen Roanne gegründet wurden, entstanden fast $90 \%$ nach 1880 und die Hälfte nach 1900. Den Großteil bildeten wie andernorts auch gesellige, nicht politische oder interessengerichtete Vereine; zusammen formten sie das sozial-moralische Rückgrat der Dritten Republik. ${ }^{50}$ In den Ländern, in denen es wenige freie Assoziation gegeben hatte, insbesondere in Österreich-Ungarn und Rußland, wird die städtische Gesellschaft zunehmend ebenfalls vom Vereinswesen geprägt. In Prag verzeichnete das Adreßbuch 1890 700, 1901 aber schon 1600 Vereine. Wie andernorts in der multiethnischen Habsburgermonarchie diente das Assoziationswesen nun dazu, Tschechen und Deutsche, Slowaken und Ungarn in jeweils eigenen Vereinen zu versammeln und sich ihrer nationalen Herkunft zu versichern. Der deutsch-jüdische Schriftsteller Paul Leppin konnte deshalb vor 1914 feststellen, daß es eine eigenständige deutsche Bevölkerung in Prag gar nicht gebe, nur eine Reihe von Vereinen. ${ }^{51}$ Wie schon im 18. und frühen 19. Jahrhundert fand ein Transfer von Ideen und sozialen Praktiken geselliger Vereine über staatliche Grenzen hinweg statt, zumeist wiederum ausgehend von England. Viktorianische Reformvereine wie z.B. die Temperance Movement erreichen nun mit ihrer Botschaft der Tugend und alkoholischen Enthaltsamkeit selbst Rußland. Die russische Gesellschaft war am Ende des Zarenreichs keine rein „staatliche Veranstaltung“ (Dietrich Geyer), sondern besaß eine öffentliche Sphäre des Gesellschaftlichen, die der zeitgenössische Begriff obshchestvennost' bezeichnete. Mehr als die Hälfte der im frühen 20. Jahrhundert gezählten 2200 Wohltätigkeitsvereine Rußlands waren nach 1890 gegründet worden. ${ }^{52} 1897$ gab es in St. Petersburg 400, 1912 in Moskau mehr als 600 verschiedene Vereine, darunter z.B. eigene Museumsgesellschaften, die gemeinhin als klassische Verkörperung des Zusammenhangs von Bürgerlichkeit und der Idee moralischer Verbesserung durch Bildung gelten. ${ }^{53}$ Der gebannte Blick auf den Staat und eine vermeintlich autoritäre Tradition hat im 20. Jahrhundert lange Zeit den Blick auf diese reiche Assoziationslandschaft Mittel- und Osteuropas vor dem Ersten Weltkrieg versperrt, wogegen in jenen Ländern, die sich in einer ungebrochenen liberalen Tradition sehen, wie z.B. Großbritannien oder den Vereinigten Staaten, den Assoziationen als Ausweis dieser besonderen Liberalität in der Historiographie große Aufmerksamkeit geschenkt wurde. ${ }^{54}$

Spannt man den zeitlichen Bogen von der Mitte des 18. bis zum frühen 20. Jahrhundert, kann man, wie gesehen, für das 18. Jahrhundert und - von West nach Ost zeitlich verschoben - für das 19. Jahrhundert die Herausbildung einer ,geselligen Gesellschaft“ innerhalb der bestehenden politischen Ordnung der kontinentaleuropäischen anciens régimes feststellen; vor 1914 sind nunmehr alle Bereiche zumindest der städtischen Gesellschaft gesellig organisiert, ungeachtet der Tatsache, daß die Mehrheit der kontinentaleuropäischen Staaten politisch nicht als Demokratien, sondern als konstitutionelle Monarchien verfaßt waren. ${ }^{55}$ Von einem Niedergang des Assoziationswesens - und

\footnotetext{
so Turner 1994, S. 4.

s1 Cohen 1981, S. 52.

52 Lindenmeyr 1996, S. 198.

53 Bradley 1990, S. 136f.

54 Eley 1992, S. 299.

55 Vgl. allg. Kirsch 1999.
} 
damit auch von „Gemeinsinn“ - in der zweiten Hälfte des 19. Jahrhunderts kann mithin keine Rede sein. Die geselligen Gesellschaften Europas und der Vereinigten Staaten beweisen vielmehr das genaue Gegenteil und verdeutlichen, daß es sich bei dem Impuls einer sozial-moralischen Verbesserung der Welt qua Assoziation um ein gemeineuropäisch-transatlantisches Phänomen handelt, das diese Gesellschaften in dem Jahrhundert vor 1914 enger miteinander verknüpft, als es der zeitgenössische Nationalismus und die Erfindung nationaler Sonderwege seit 1914 vermuten läßt. ${ }^{56}$

Zeugt die Verbreitung des Assoziationsprinzips von den Erfolgen liberaler Ideen und Praktiken, mehren sich nun aber die kritischen Stimmen zur „Vereinsmeierei“ der Zeit - insbesondere von liberaler Seite. Die moralische Utopie einer Verbesserung des Menschen, seiner Erziehung zu Tugend und Gemeinsinn im geselligen Austausch mit anderen, schien angesichts der Verallgemeinerung und zahlenmäßigen Explosion der Vereine weniger glaubhaft. Je mehr sich das Vereinswesen ausweitete und auch jene zuvor ausgeschlossenen Gruppen erfaßte, desto mehr schien dieser Anspruch und mit ihm das Vertrauen in die Macht der Tugend und Versittlichung überzogen. Nicht das Assoziationswesen, sondern die mit ihm verbundene politisch-moralische Gesellschaftsvision, die Tocqueville eindringlich formuliert hatte, befand sich am Ende des Jahrhunderts in der Krise.

Diese Desintegration und Pluralisierung des Anspruchs auf Tugend, Geselligkeit und moralische Verbesserung war aber ein Resultat der Demokratisierung, nicht ihr Gegenteil. Aus den Arbeitervereinen, die in Großbritannien und Deutschland zunächst der sozialen Reform und moralischen „Verbesserung“ der arbeitenden Bevölkerung unter der Aufsicht liberaler Bürger dienten, enstand in den 1880er Jahren eine eigene gesellige Gegenkultur, die sich dieser Aufsicht ebenso entzog wie die neuen klassenübergreifende Kulturformen der frühen Massenkultur. ${ }^{57}$ Ähnlich verhält es sich mit dem aus der zeitgenössischen Sicht wichtigsten Gegner des Liberalismus, dem europäischen Katholizismus, der sich - strukturell ähnlich wie die Arbeiterbewegung - der geselligen Vereine zur sozial-moralischen Milieubildung bis hinein in die ländliche Bevölkerung als Schutz gegen den Zugriff des säkularisierten Staates bediente. Nicht nur Besitz und Bildung, sondern auch Konfession und Geschlecht bestimmten die Zugehörigkeit zu geselligen Vereinen vor ihrer Demokratisierung gegen Ende des 19. Jahrhunderts. Bis dahin gehörte der Ausschluß von Frauen ebenso selbstverständlich zu den Praktiken der "geselligen Gesellschaft" wie die Gleichsetzung von Bürgertugend und Männlichkeit. Jetzt gründeten Frauen eigene Vereine, die sozial-moralische Ansprüche etwa im Bereich der Wohltätigkeit und Sozialfürsorge erhoben - auch das ein transnationales Phänomen. Juden, denen die gleichberechtigte Teilhabe am allgemeinen Vereinswesen in Mitteleuropa lange Zeit verwehrt blieb, fanden nun Zugang oder bildeten eigene Geselligkeitsformen, etwa logenähnliche Gesellschaften wie den Orden „B'nai Brith“ - gegründet von deutsch-jüdischen Emigranten 1843 in New York und von dort seit den achtziger Jahren sich auf dem europäischen Kontinent verbreitend.

Welche Bevölkerungsgruppe vom ,respektablen" bürgerliche Vereinsleben einer lokalen Gesellschaft ausgeschlossen blieb, variierte jeweils nach politisch-sozialem Kon-

\footnotetext{
56 Vgl. hierzu exemplarisch Rodgers 1998.

57 Vgl. u.a. Price 1971; Lidtke 1985.
} 
text. Kaum eine Grenze aber wurde schärfer gezogenen als die der weißen amerikanischen Mittelklassen und ihrer geselligen Vereine gegenüber den Afro-Amerikanern, gerade gegenüber den freien und „respektablen“, die ensprechend frühzeitig eigene Assoziationen und geheime Gesellschaften gründeten. „No respectability“, schrieb ein englischer Besucher Philadelphias im Jahr 1818, „however unquestionable, - no property, however large, - no character, however unblemished, - will gain a man, whose body is (in American estimation) cursed with even a twentieth portion of the blood of his African ancestry, admission into Society“ ${ }^{58}{ }^{58}$ Entsprechend suchten die Afro-Amerikaner in Philadelphia wie andernorts mit ihren Logen und Vereine die der weißen Mittelklassen an Tugendhaftigkeit und Gemeinsinn noch zu übertreffen. Die Gleichzeitigkeit von sozialem Ausschluß und einer zunehmenden Konkurrenz in den moralischen Ansprüchen hat in all den hier betrachteten Gesellschaften im Laufe des 19. Jahrhunderts die Verbreitung der geselligen Vereine befördert und zugleich den Glauben an ihre politisch-moralische Bedeutung in Zweifel gezogen.

Der Zusammenhang zwischen Assoziationsprinzip und dem Anspruch, das Allgemeine Beste zu vertreten, geriet zusätzlich unter Druck aufgrund eines weiteren zwiespältigen liberalen Erfolges: des Aufstiegs der Nation als politischer Ordnungsidee. Der Anspruch auf das gemeine Wohl war schon in der Spätaufklärung und im Frühliberalismus gebunden an ein Bekenntnis zur Nation. Für Tocqueville sollte das Vaterland das größte und engste Band knüpfen, das die Menschen in der Demokratie zusammenhält. Und für Welcker, um bei dem vorn zitierten Lexikonartikel zu bleiben, galt 1846 der Tod für das Vaterland als die höchste Bürgertugend - eine Forderung, die erst im Zeitalter nationaler Kriege ihre wahre Bedeutung entfalten sollte. ${ }^{59}$ Der Nationalismus des 19. Jahrhunderts organisierte sich bekanntlich in geselligen Vereinen. In dem Maße, wie sich die "geselligen Gesellschaften" in Nationalgesellschaften umbildeten, zeugte die Berufung auf die Nation mehr von den Konflikten innerhalb wie zwischen den Staaten als von einem abstrakten allgemeinen Wohl. In den universalen Anspruch auf die gesellige Aneignung von Tugend und Gemeinsinn mischten sich mithin von Anbeginn soziale, religiöse und politische Forderungen, die wiederum partikularen Interessen folgen konnten. Paradoxerweise beruhte die Universalität des Assoziationsprinzips, seine Erfolgsgeschichte im Jahrhundert vor 1914 auf dieser Verknüpfung von universalen Ansprüchen und partikularen Interessen. Die Vereine können sozialgeschichtlich gesehen als das wichtigste Medium für die Herausbildung und Verfestigung neuer Identitäten im 19. Jahrhundert gelten, seien sie nationaler oder sozialer, konfessioneller oder politischer Art, die jeweils eigene Ansprüche erhoben. Die Pluralisierung der sozialen Praktiken der "geselligen Gesellschaft" hatte die Fragmentierung ihres politischmoralischen Anspruchs auf Tugend und Gemeinsinn zur Folge.

In dem Moment, wo die liberalen Bürger in den Vereinen nicht mehr in dem Bewußtsein leben konnten, das allgemeine Wohl allein zu vertreten, mehren sich nicht nur Zweifel an den Ideen, sondern auch an den Praktiken des Liberalismus, etwa an der politischen Bedeutung der geselligen Vereine. Daß in Frankreich (1901) und Deutschland (1908) erst vergleichsweise spät die Vereinsfreiheit ohne jede Einschränkung ge-

58 Nash 1988 , S. 226.

59 Welcker 1846, S. 751. Vgl. Nägler 1990; Meyer 1994; sowie die Hinweise bei Münkler 1991. 
setzlich verankert wurde, ist weniger ein Ergebnis des vermeintlich autoritären Charakters des Staates als der Angst der Bürger vor den in ihren Augen unbürgerlichen Mächten der Gesellschaft, z.B. den Katholiken und Sozialisten. ${ }^{60}$ Die wildwüchsige Ausweitung des Assoziationswesens in alle Schichten der Gesellschaft hinein ging mit einer zunehmenden Furcht bürgerlicher Kreise vor einem Verlust des moralischen Führungsanspruchs in der Gesellschaft einher, den bis dahin das Assoziationsprinzip verbürgt hatte.

So formulierte Max Weber mit indigniertem Unterton auf dem Ersten Deutschen Soziologentag im Jahr 1910, daß der moderne, „letzte“ Mensch „ein Vereinsmensch in einem fürchterlichen, nie geahnten Maße" sei. „Man muß ja glauben: das ist nicht mehr zu überbieten, seitdem sich auch ,Vereins-Enthebungs'-Organisationen gebildet haben." ${ }^{\text {"61 }}$ Dennoch ist gerade in den entstehenden Sozial- und Kulturwissenschaften der Jahrhundertwende ein Bewußtsein für den Stellenwert geselliger Vereine noch lebendig gewesen, das sich erst in der Zwischenkriegszeit verlieren sollte. Gerade aus dem Krisengefühl der Jahrhundertwende heraus gewinnt der Zusammenhang von Sozialität und Tugend der klassischen politischen Theorie etwa für Weber noch einmal jene leidenschaftliche politische Bedeutung, wie sie Tocqueville eindringlich formuliert hatte. Auch Weber ging es nicht allein um eine Analyse der modernen, nach rationalen Interessen organisierten Gesellschaft, sondern um ihre Auswirkung auf die „Seelenverfassung“ der von ihr geprägten Individuen, ihr „Menschentum“. „Nicht die Förderung des Kapitalismus in seiner Expansion“, hält etwa Weber den Kritikern seiner „Protestantische Ethik" entgegen, „war das, was mich zentral interessierte, sondern die Entwicklung des Menschentums, welches durch das Zusammentreffen religiös und ökonomisch bedingter Komponenten geschaffen wurde", ein bestimmter „ethischer Lebensstil", wie er an anderer Stelle schreibt, „welcher der Wirtschaftsstufe des ,Kapitalismus' geistig ,adäquat" war [und der] seinen Sieg in der ,Seele ${ }^{6}$ des Menschen bedeutete. ${ }^{662}$

Tocquevilles Fragestellung aufnehmend, sah Weber nicht allein in der Ausbreitung, Verflechtung und Zusammensetzung von Assoziationen, sondern in der „Frage nach der Beeinflussung des menschlichen Gesamthabitus durch die verschiedenen Inhalte der Vereinstätigkeit" den Schlüssel zum politischen Verständnis der Geselligkeit. ${ }^{63}$ „Wie wirkt die Zugehörigkeit zu einem bestimmten Verband nach innen?“ fragt Weber, ,auf die Persönlichkeit als solche? [...] Welches spezifische Ideal von ,Männlichkeit‘ [wird], bewußt oder absichtsvoll oder auch unbewußt ... gepflegt?" „Welche Art von Beziehung besteht zwischen einem Verein irgendwelcher Art, wieder von der Partei bis - das klingt ja paradox - zum Kegelklub herab, zwischen einem beliebigen Verein und irgend etwas, was man, im weitesten Sinne des Wortes Weltanschauung nennen kann?" ${ }^{64}$ Mit anderen Worten: Welchen Menschentypus bringt die gesellige Gesellschaft hervor?

\footnotetext{
${ }^{60}$ Harrison 1999, S. 33.

61 Weber 1911, S. 53.

62 Weber 1978, S. 303 u. 55, Anm. 5, zit. n. Hennis 1996, S. 44.

${ }^{63}$ Zit. n. Weber (1926) 1989, S. 428; ähnlich auch Weber 1911, S. 58. Vgl. hierzu Hennis 1987; Hecht 1998, S. 199-250.

${ }^{64}$ Weber 1911, S. 55.
} 
In der eingeschobenen Reserve gegenüber den gewöhnlichen Kegelklubs, die für Putnam heute symptomatische Bedeutung haben, drückt Weber aber zugleich die eigenen Zweifel aus, ob die Evokation des Zusammenhangs von Tugend und Geselligkeit für seine Zeit noch Gültigkeit besitzt. Schließlich sei es „eine alltägliche Erscheinung, daß Vereinigungen, die ausgegangen sind von großen Weltanschauungsideen, zu Mechanismen werden, die sich faktisch davon loslösen.“ Das liege in der „,Tragik“ jedes Realisationsversuchs von Ideen in der Wirklichkeit überhaupt“. Und weiter: „Es gehört ja zu jedem Verein bereits ein, sei es bescheidener Apparat, und sobald der Verein propagandistisch auftritt, wird dieser Apparat in irgend einer Weise versachlicht und vom Berufsmenschentum okkupiert." Es ist dieses Berufsmenschentum, das für Weber die politische Tugend aufzehrt und das künftig im Gehäuse der kapitalistischen Lebensordnung wohnen könnte. Am Schluß der „Protestantischen Ethik“ hat er es leidenschaftlich beschrieben: Es sind jene „letzten Menschen“, die auch Nietzsche heraufkommen sah, die „Fachmenschen ohne Geist, Genußmenschen ohne Herz". ${ }^{65}$ Wie Nietzsche stellt sich auch Weber mit Blick auf die modernen Lebensordnungen das Problem, „was wir dieser Maschinerie entgegenzusetzen haben, um einen Rest des Menschentums frei zu halten von dieser Parzellierung der Seele, von dieser Alleinherrschaft bürokratischer Lebensideale". ${ }^{66}$

War sich Weber zumindest unsicher, ob die Vereine Agenten oder Verhinderer dieser politisch gefährlichen „Parzellierung der Seele“ sind, hatte Nietzsche für den liberalen Glauben an einem Zusammenhang von Geselligkeit und Tugend nur Hohn und Spott übrig. Der nicht nur in der sozialen Ausweitung des Assoziationswesen erkennbaren Tendenz zur Demokratisierung setzte er einen aristokratischen Tugendbegriff entgegen. Während die „letzten" Menschen sich wie Herdentiere Zusammenschließen in ,geselligen Gesellschaften" und „demokratischen Vaterländern“, verlieren sie die wahre politische Tugend, die der Abschließung und individuellen Aneignung bedarf. Im Zeitalter der massenhaften Ausweitung geselliger Vereine, der „Spießbürger“ und „Vereinsmeier“, bleibt für Nietzsche nur eine Tugend übrig: die Einsamkeit. „Denn die Einsamkeit ist bei uns eine Tugend, als ein sublimer Hang und Drang der Reinlichkeit, welcher erräth, wie es bei Berührung von Mensch zu Mensch - in Gesellschaft ${ }^{*}$ - unvermeidlich-unreinlich zugehn muß." Nichts schien Nietzsche nun gegen Ende des 19. Jahrhunderts absurder als die Vorstellung seiner vereinsseligen Zeitgenossen, der gemeinsame Zusammenschluß erzeuge politische Tugend und Gemeinsinn: „Jede Gemeinschaft macht, irgendwie, irgendwo, irgendwann - gemein'.667

Die Berührung von Mensch zu Mensch in Gesellschaft galt Tocqueville zeitlebens als einziges Mittel, einen heraufziehenden Despotismus und seinen Sieg in der "Seele“ der Menschen zu verhindern. Der Despotismus mauere die Menschen im Privatleben ein. „Sie waren bereits zur Absonderung geneigt: er isoliert sie; sie erkalten füreinander: er läßt sie vollends erstarren. " ${ }^{68}$ Wie politisch zeitgemäß ein solcher Glauben an einen Zusammenhang von Tugend und Geselligkeit auch sein mag, seine historischen Folgen

${ }^{65}$ Weber, 1988, 204.

${ }^{66}$ Weber 1924, S. 413.

${ }^{67}$ Nietzsche [1885] 1988, S. 232. Vgl. hierzu Baier 1981/82.

68 Tocqueville [1856] 1978, S. 15. 
waren zumindest ambivalent. Der Gemeinwohlanspruch, der sich auf das Bewußtsein stützte, eine Elite, die sich in geselligen Vereinen ihrer „Qualität" versicherte, zu vertreten, war immer schon verbunden mit sozialen oder moralischen, nationalen oder ,rassischen", religiösen oder geschlechtsspezifischen Vorannahmen. Zu den Leidenschaften der geselligen Bürger des 19. Jahrhunderts gehörte, wie Philip Nord betont, nicht nur der Wille, für das Allgemeine Beste zu wirken, sondern auch der Ausschluß, die Maßregelung und politisch-moralische Erziehung jener, die nicht den bürgerlichen Normen entsprachen. ${ }^{69}$ Die Ballotage in einem geselligen Verein war im 19. Jahrhundert nicht nur eine Einübung in demokratische Praktiken, sondern ein Ausschlußmechanismus jener, die bestimmten sozial-moralischen Ansprüchen nicht genügten. Tocquevilles These eines Zusammenhangs von Demokratie und Assoziation, an die heute die Freunde der Bürgergesellschaft anknüpfen wollen, muß folglich historisch relativiert werden. Wie Assoziationen selbst verfaßt waren, welche sozial-moralischen Ansprüche sie formulierten und welche mitunter gegenläufigen Resultate diese Ansprüche zeitigten, muß genauer in den Blick genommen werden.

\section{Literatur:}

Agulhon, M. (1977), Le cercle dans la France bourgeoisie 1810-1848. Etude d'une mutation de sociabilité, Paris.

Agulhon, M. (1984), Pénitents et Francs Maçons de l'ancienne Province, Paris.

Appleby, J. (1992), Liberalism and Republicanism in the Historical Imagination, Cambridge, Mass.

Aron, R. (1981), Alexis de Tocqueville und Karl Marx, in: ders., Über die Freiheiten, Stuttgart, S. 13-45.

Baier, H. (1981/82), Die Gesellschaft - ein langer Schatten des toten Gottes. Friedrich Nietzsche und die Entstehung der Soziologie aus dem Geiste der Décadence, in: Nietzsche-Studien, Bd. 10/11, 6-33.

Blumin, St. M. (1989), The Emergence of the Middle Class. Social Experience in the American City, 1760-1900, Cambridge, Mass.

Bradley, J. (1990), Voluntary Associations, Civic Culture, and Obshchestvennost' in Moscow, in: Between Tsar and People. Educated Society and the Quest for Public Identity in Late Imperial Russia, hg. v. E. W. Clowes u.a., Princeton, S. 131-48.

Bullock, St. C. (1996), Revolutionary Brotherhood. Freemasonry and the Transformation of the Amercian Social Order, 1730-1840, Chapel Hill.

Caglioti, D. L. (1996), Associazionismo e sociabilità d'élite a Napoli nel XIX secolo, Neapel.

Carnes, M. C. (1989), Secret Ritual and Manhood in Victorian America, New Haven.

Clawson, M. A. (1989), Constructing Brotherhood. Class, Gender and Fraternalism, Princeton

Dumenil, L. (1984), Freemasonry and American Culture, 1880-1930, Princeton.

Clark, P. (2000), British Clubs and Societies 1580-1800. The Origins of an Associational World, New York.

Cohen, G. (1981), The Politics of Ethnic Survival. Germans in Prague, 1861-1914, Princeton.

Eley, G. (1992), Nations, Publics, and Political Cultures. Placing Habermas in the Nineteenth Century, in: Habermas and the Public Sphere, hg. v. Cr. Calhoun, Cambridge, Mass., S. 289-339.

\footnotetext{
${ }^{69}$ Nord 2000; ähnlich auch Harrison 1996; Trentmann 2000.
} 
François, E. (Hg.) (1986), Sociabilité et société bourgeoise en France, en Allemagne et en Suisse, 1750-1850, Paris.

Furet, Fr. (1980), 1789 - Vom Ereignis zum Gegenstand der Geschichtswissenschaft, Frankfurt/M.

Gall, L. (1976), Liberalismus und „bürgerliche Gesellschaft“. Zu Charakter und Entwicklung der liberalen Bewegung in Deutschland, in: Liberalismus, hg. v. Ders., Köln, 162-86.

Gilkeson Jr., J. S. (1986), Middle-Class Providence, 1820-1940, Princeton 1986.

Goltermann, S. (1998), Körper der Nation. Habitusformierung und die Politik des Turnens 1860-1890, Göttingen.

Gordon, D. (1994), Citizens without Sovereignity. Equality and Sociability in French Thought, 1670 1789 , Princeton.

Hardtwig, W. (1984), Strukturmerkmale und Entwicklungstendenzen des Vereinswesens in Deutschland 1789-1848, in: Vereinswesen und bürgerliche Gesellschaft in Deutschland, hg. v. O. Dann, München, S. 11-50.

Harrison, C. E. (1996), Unsociable Frenchmen. Associations and Democracy in Historical Perspective, in: The Tocqueville Review, Jg. 17, 37-56.

Harrison, C. E. (1999), The Bourgeois Citizen in Nineteenth-Century France. Gender, Sociability, and the Uses of Emulation, Oxford.

Hausmann, G. (2000), Die wohlhabenden Odessaer Kaufleute und Unternehmer. Zur Herausbildung bürgerlicher Identitäten im ausgehenden Zarenreich, in: Jahrbücher für die Geschichte Osteuropas, Jg. 48, S. 41-65.

Hecht, M. (1998), Modernität und Bürgerlichkeit. Max Webers Freiheitslehre im Vergleich mit den politischen Ideen von Alexis de Tocqueville und Jean-Jacques Rousseau, Berlin.

Hein, D./A. Schulz (Hg.) (1996): Bürgerkultur im 19. Jahrhundert. Bildung, Kunst und Lebenswelt, München.

Hennis, W. (1982), Tocquevilles ,Neue Politische Wissenschaft', in: Aspekte der Kultursoziologie, hg. v. J. Stagl, Berlin, S. 385-407.

Hennis, W. (1987), Max Webers Fragestellung, Tübingen.

Hennis, W. (1996), Max Webers Wissenschaft vom Menschen, Tübingen.

Hoffmann, St.-L. (2000), Die Politik der Geselligkeit. Freimaurerlogen in der deutschen Bürgergesellschaft, 1840-1918, Göttingen.

Howe, D. W. (1997), Making the American Self. Jonathan Edwards to Abraham Lincoln, Cambridge, Mass.

Hull, I. V. (1995), Sexuality, State, and Civil Society in Germany 1700-1815, Ithaca 1995.

Jardin, A. (1991), Alexis de Tocqueville. Leben und Werk, Frankfurt/M.

Jacob, M. C. (1991), Living the Enlightenment. Freemasonry and Politics in Eighteenth-Century Europe, New York.

Janowski, M. (2000), Marginal or Central? The Place of Liberal Tradition in the Polish 19th Century History (MS).

Jedlinski, J. (1999), A Suburb of Europe. Nineteenth-Century Polish Approaches to Western Civilization, Budapest.

Kahan, A. (1992), Aristocratic Liberalism. The Social and Political Thought of Jacob Burckhardt, John Stuart Mill, and Alexis de Tocqueville, New York.

Kirsch, M. (1999), Monarch und Parlament. Der monarchische Konstitutionalismus als europäischer Verfassungstyp, Göttingen. 
Klein, L. E. (1998), Sociability, Solitude and Enthusiasm, in: ders. u. A. J. La Vopa (Hg.), Enthusiasm and Enlightenment in Europe, 1650-1850 (= Huntington Library Quarterly, Jg. 60, H. 1/2), Oxford, S. 153-77.

Klein, L. E. (1994), Shaftesbury and the Culture of Politeness. Moral Discourse and Cultural Politics in Early Eighteenth-Century England, Cambridge.

Kloppenberg, J. T. (1996), Life Everlasting: Tocqueville in America, in: The Tocqueville Review, Jg. 17, S. 19-36.

Koselleck, R. (1989), Kritik und Krise. Eine Studie zur Pathogenese der bürgerlichen Welt [1959], Frankfurt/M.

Koselleck, R. u.a. (1991), Drei bürgerliche Welten? Zur vergleichenden Semantik der bürgerlichen Gesellschaft in Deutschland, England und Frankreich, in: Bürger in der Gesellschaft der Neuzeit, hg. v. H.-J. Puhle, Göttingen, S. 14-58.

Koselleck, R./K. Schreiner (Hg.) (1994), Bürgerschaft. Rezeption und Innovation der Begrifflichkeit vom Hohen Mittelalter bis ins 19. Jahrhundert, Stuttgart.

Koselleck, R. (o.J.), Liberales Geschichtsdenken, in: Liberalismus - nach wie vor. Grundgedanken und Zukunftsfragen, Zürich, S. 29-51.

Langewiesche, D. (1997), Frühliberalismus und Bürgertum 1815-1849, in: Bürgertum und bürgerlichliberale Bewegung in Mitteleuropa seit dem 18. Jahrhundert, hg. v. L. Gall, München, S. 63-129.

Lidtke, V. (1985), The Alternative Culture. Socialist Labor in Imperial Germany, New York.

Lindenmeyr, A. (1996), Poverty Is Not a Vice. Charity, Society, and the State in Imperial Russia, Princeton.

Mannová, E. (1998), Middle-Class Identities in a Multicultural City. Associations in Bratislava in the 19th Century. Paper given at the Fourth International Conference on Urban History: Cities in Europe. Places and Institutions, Venice, September 3-5.

Marshall, L. L./S. Drescher (1968), American Historians and Tocqueville's Democracy, in: Journal of American History, Jg. 55, S. 512-32.

Meriggi, M. (1992), Milano borghese. Circoli ed élites nell'Ottocento, Venedig.

Mettele, G. (1998), Bürgertum in Köln 1775-1870. Gemeinsinn und freie Assoziation, München.

Meyer, M. (1994), Freiheit und Macht. Studien zum Nationalismus süddeutscher, insbesondere badischer Liberaler 1830-1848, Frankfurt/M.

Münkler, H. (1996), Einleitung, in: Ders. (Hg.), Bürgerreligion und Bürgertugend. Debatten über vorpolitische Grundlagen politischer Ordnung, Baden-Baden, S. 7-11.

Münkler, H. (1991), Die Idee der Tugend. Ein politischer Leitbegriff im vorrevolutionären Europa, in: Archiv für Kulturgeschichte, Bd. 73, S. 379-403.

Münkler, H. (1992), Politische Tugend. Bedarf die Demokratie einer sozio-moralischen Grundlegung?, in: Ders. (Hg.), Die Chancen der Freiheit, München, S. 25-46.

Mullan, J. (1988), Sentiment and Sociability. The Language of Feeling in the Eighteenth Century, Oxford.

Nägler, Fr. (1990), Von der Idee des Friedens zur Apologie des Krieges. Eine Untersuchung geistiger Strömungen im Umkreis des Rotteck-Welckerschen Staatslexikons, Baden-Baden.

Nash, G. B. (1988), Forging Freedom. The Formation of Philadelphia's Black Community 1720-1840, Cambridge, Mass.

Nietzsche, Fr. (1988), Jenseits von Gut und Böse [1885], in: Kritische Studienausgabe, hg. v. G. Colli/M. Montinari, Bd. 5, München. 
Nipperdey, Th. (1976), Verein als soziale Struktur in Deutschland im späten 18. und frühen 19. Jahrhundert., in: ders., Gesellschaft, Kultur, Theorie, Göttingen, S. 174-205.

Nolte, P. (1992a), Bürgerideal, Gemeinde und Republik. „Klassischer Republikanismus“ im frühen deutschen Liberalismus, in: Historische Zeitschrift, Bd. 254, S. 609-56.

Nolte, P. (1992b), Der südwestdeutsche Liberalismus in der Kontinuität der Frühen Neuzeit, in: Geschichte in Wissenschaft und Unterricht, Jg. 43, S. 743-56.

Nolte, P. (1994), Gemeindebürgertum und Liberalismus in Baden 1800-1850, Göttingen.

Nord, Ph. (1991), Republicanism and Utopian Vision. French Freemasonry in the 1860s and 1870s, in: Journal of Modern History, Jg. 63, S. 213-29.

Nord, Ph. (1995), The Republican Moment. Struggles For Democracy in Nineteenth-Century France, Cambridge, Mass.

Nord, Ph. (2000), Introduction, in: ders. u. Nancy Bermeo (Hg.), Civil Society Before Democracy. Lessons from Nineteenth-Century Europe, Boston, S. xiii-xxxiii.

Pocock, J. G. A. (1975), The Machiavellian Moment. Florentine Political Thought and the Atlantic Republican Tradition, Princeton.

Pocock, J. G. A. (1989), Civic Humanism and its Role in Anglo-American Thought, in: Ders., Politics, Language, and Time. Essays on Political Thought and History, Chicago, 80-103.

Price, R. N. (1971), The Working Men's Club Movement and Victorian Social Reform Ideology, in: Victorian Studies, Jg. 15, S. 117-47.

Putnam, R. D. (1995), Bowling Alone. America's Declining Social Capital, in: Journal of Democracy, Jg. 6, S. 65-78.

Putnam, R. D. (1996), The Strange Disappearance of Civic America, in: The American Prospect, Nr. 24, S. 34-48.

Putnam, R. D. (2000), Bowling Alone. The Collapse and Revival of American Community, New York.

Putnam, R. D. (1993), Making Democracy Work. Civic Traditions in Modern Italy, Princeton.

Reddy, W. (1997), The Invisible Code. Honor and Sentiment in Postrevolutionary France, 1814-1848, Berkeley.

Roche, D. (1981), Die sociétés de pensée und die aufgeklärten Eliten im 18. Jahrhundert, in: Sozialgeschichte der Aufklärung in Frankreich, hg. v. R. Reichardt/H.-U. Gumbrecht, München, S. 77-115.

Rodgers, D. T. (1992), Republicanism. The Career of a Concept, in: Journal of American History, Jg. 79, S. 1-38.

Rodgers, D. T. (1998), Atlantic Crossings. Social Politics in a Progressive Age, Cambridge, Mass.

Rotteck, C. v. (1838), Gemeingeist oder Gemeinsinn, in: Das Staatslexikon. Encyklopädie der sämtlichen Staatswissenschaften für alle Stände, hg. v. C. v. Rotteck/C. Th. Welcker, Bd. 1-15, Bd. 6, Altona, S. 448-59.

Ryan, M. P. (1981), Cradle of the Middle Class. The Family in Oneida County, New York, 17901865, Cambridge.

Ryan, M. P. (1997), Civic Wars. Democracy and Public Life in the American City During the Nineteenth-Century, Berkeley.

Skocpol, Th./M. Fioring (Hg.) (1999), Civic Engagement in American Democracy, Washington, D.C.

Skocpol, Th. (1997), The Tocqueville Problem. Civic Engagement in American Democracy, in: Social Science History, Jg. 21, S. 455-79.

Smith, D. (1995), Freemasonry and the Public in Eighteenth-Century Russia, in: Eighteenth-Century Studies, Jg. 29, S. 25-44. 
Smith, D. (1999), Working the Rough Stone. Freemasonry and Society in Eighteenth-Century Russia, DeKalb.

Stekl, H. u.a. (Hg.) (1992), Bürgertum in der Habsburgermonarchie, Bd. 2: „Durch Arbeit, Besitz, Wissen und Gerechtigkeit", Wien.

Steinmetz, W. (1991), Die schwierige Selbstbehauptung des deutschen Bürgertums, in: Das 19. Jahrhundert, hg. v. R. Wimmer, Berlin, S. 12-40.

Tenfelde, K. (1984), Die Entfaltung des Vereinswesens während der industriellen Revolution in Deutschland (1850-1873), in: Vereinswesen und bürgerliche Gesellschaft in Deutschland, hg. v. O. Dann, München, S. 55-114.

Tocqueville, Al. de (1987), Über die Demokratie in Amerika, T. 1 [1835], Zürich.

Tocqueville, Al. de (1987): Über die Demokratie in Amerika, T. 2 [1840], Zürich.

Tocqueville, Al. de (1978): Der alte Staat und die Revolution [1856], München.

Trentmann, Fr. (2000), Introduction, in: hg. v. Ders., Paradoxes of Civil Society. New Perspectives on Modern German and British History, Providence, S. 3-45.

Turner, P. R. (1994), Class, Community and Culture in Nineteenth Century France. The Growth of Voluntary Associations in Roanne, 1860-1914, Ph.D. diss. University of Michigan, Ann Arbor.

Vincent-Buffault, A. (1995), L'Exercice de l'amitié: pour une histoire des pratiques amicales aux XVIIIe et XIXe siècles, Paris.

Wahrman, D. (1995), Imagining the Middle-Class. The Political Representation of Class in Britain, c. 1780-1840, Cambridge.

Weber, M. (1989), Max Weber, Ein Lebensbild [1926], München.

Weber, M. (1911), Geschäftsbericht, in: Verhandlungen des Ersten Deutschen Soziologentages vom 19. bis 22. Okt. 1910 in Frankfurt/M., Tübingen, S. 52-62.

Weber, M. (1924), Gesammelte Aufsätze zur Soziologie und Sozialpolitik, Tübingen.

Weber M. (1978), Die protestantische Ethik II. Kritiken und Antikritiken, hg. v. J. Winckelmann, Gütersloh.

Weber, M. (1988), Gesammelte Aufsätze zur Religionssoziologie, Bd. 1, Tübingen.

Welcker, C. Th. (1835), Association, Verein, Gesellschaft, Volksversammlung, in: Das Staatslexikon. Encyklopädie der sämtlichen Staatswissenschaften für alle Stände, Bd. 1-15, Altona, Bd. 2, S. 21-53.

Welcker, C. Th. (1846), Bürgertugend und Bürgersinn, in: Das Staatslexikon. Encyklopädie der sämtlichen Staatswissenschaften für alle Stände, Bd. 1-15, 1. Suppl. Bd., Altona, S. 748-58.

Wilentz, S. (1988), Many Democracies. On Tocqueville and Jacksonian America, in: Reconsidering Tocqueville's Democracy in America, hg. v. A. S. Eisenstadt, New Brunswick, S. 207-28.

Wirsching, A. (1990), Bürgertugend und Gemeininteresse. Zum Topos der „Mittelklassen“ in England im späten 18. und frühen 19. Jahrhundert, in: Archiv für Kulturgeschichte, Bd. 72, S. 173-99.

Wood, G. S. (1999), The American Love Boat (Rezension von: Andrew Burstein, Sentimental Democracy. The Evolution of America's Romantic Self-Image, New York 1999), in: New York Review of Books, 7. Okt.

Wood, G. S. (1999), The Radicalism of the American Revolution, New York. 
